\title{
Use of perfluorocarbon liquids in proliferative vitreoretinopathy: results and complications
}

University of Vienna, Department of Ophthalmology B, Vienna, Austria U Stolba $S$ Binder $M$ Velikay P Datlinger A Wedrich

Correspondence to: Ulrike Stolba, MD, University of Vienna, Department of Ophthalmology B Währinger Gürtel 18-20, 1090 Vienna, Austria. 1090 Vienna, Austria. 13 September 1995

\begin{abstract}
Background-The present study was set up to evaluate the influence of perfluorocarbon liquids on the postoperative anatomical and functional results as well as on the complication rates in eyes with proliferative vitreoretinopathy (PVR).

Methods-Sixty five consecutive eyes (64 patients) with PVR in different stages requiring surgical intervention where liquid perfluorocarbons were used were compared with 64 consecutive eyes (62 patients) operated without the help of perfluorocarbon immediately before this time. Both groups were similar with regard to severity of PVR, number of operations, and initial visual acuity. The observation period was shorter in the perfluorocarbon group because they were operated more recently $(17.4$ months as against 24.4 months).
\end{abstract}

Results-The anatomical as well as the functional success rates were not significantly higher in the perfluorocarbon group $(69 \% v 61 \%$ and $65 \%$ v 53\% respectively). However, in cases operated on without perfluorocarbons where reproliferation would occur it was of much greater severity than in cases where perfluorocarbons were used. The number of uncured cases with contraction of the retina at least in the inferior half was more than twice as high in the group operated on without perfluorocarbon. Combined with massive reproliferation secondary glaucoma and bullous or band keratopathy were more frequent in eyes treated before the use of perfluorocarbon. Conclusion-The intraoperative use of perfluorocarbon liquids in vitreoretinal surgery does not prevent postoperative reproliferation but does reduce its severity. (Br f Ophthalmol 1995; 79: 1106-1110)

Since liquid fluorocarbons were introduced by Chang in 1987 for intraoperative use in eyes with giant retinal tears or severe proliferative vitreoretinopathy $(P V R)^{1}$ there is general agreement that these heavy liquids are a new surgical approach to facilitate and improve vitreoretinal techniques. ${ }^{2}$ The indication for their application has been expanded to PVR after ocular trauma and to eyes with subluxation of the lens into the vitreous cavity.

To determine the effect of intraoperative perfluorocarbons on the final anatomical and functional results we compared retrospectively two similar groups of eyes with PVR due to aphakia, pseudophakia, or uncured idiopathic retinal detachment, myopia, blunt or perforating injuries without intraocular foreign bodies.

\section{Materials and methods}

A total of 126 patients (129 eyes) (53 females and 73 males) aged between 10 and 85 years (average 57.3 years) were included in the study.

We reviewed the records of 64 consecutive patients and 65 eyes ( 28 females, 36 males aged 16 to 81 years, average 58.4 years) with PVR retinal detachment operated on with the help of perfluorocarbons between May 1991 and October 1992 (group 1). Indications for surgery, distribution of the PVR grades according to the classification of Machemer $1991,{ }^{3}$ and initial visual acuities are listed in Tables 1-3.

We compared the anatomical results of this group with 64 consecutive PVR cases of 62 patients (25 females and 37 males aged between 10 and 85 years, average $56 \cdot 2$ years, group 2) where surgery was performed before the routine use of perfluorocarbons (May 1989 to October 1991). In this group the indications for surgery as well as the PVR grades are comparable with the first group (see Tables 1 and 2).

Excluded from the study were patients who

Table 1 Indication for surgery

\begin{tabular}{llllll}
\hline & \multicolumn{2}{l}{ Group 1 } & & \multicolumn{2}{c}{ Group 2 } \\
\cline { 2 - 3 } \cline { 5 - 6 } & $n$ & $(\%)$ & & $n$ & $(\%)$ \\
\hline Aphakic or pseudophakic RD & 20 & $(31)$ & & 22 & $(34 \cdot 5)$ \\
Myopia & 18 & $(28)$ & & 20 & $(31)$ \\
Idiopathic PVR & 16 & $(24 \cdot 5)$ & & 12 & $(19)$ \\
Blunt trauma & 4 & $(6)$ & 4 & $(6)$ \\
Perforation injury & 7 & $(10 \cdot 5)$ & 6 & $(9 \cdot 5)$ \\
\hline
\end{tabular}

$\mathrm{RD}=$ retinal detachment; $\mathrm{PVR}=$ proliferative vitreoretinopathy.

Table 2 Distribution of proliferative vitreoretinopathy (PVR) grades

\begin{tabular}{|c|c|c|c|c|}
\hline \multirow[b]{2}{*}{ PVR grades } & \multicolumn{2}{|c|}{ Group 1} & \multicolumn{2}{|c|}{ Group 2} \\
\hline & $n$ & $(\%)$ & $n$ & $(\%)$ \\
\hline $\begin{array}{l}\text { C Ant } 1-6^{\star} \\
\text { C Ant } 1-6^{\star} \text { Post } 1-6^{\star} \\
\text { C Ant } 1-12^{\star} \text { Post } 1-12^{\star} \\
\text { C Post } 1-6^{\star} \\
\text { C Post or Ant } 1-12^{\star}\end{array}$ & $\begin{array}{r}3 \\
28 \\
25 \\
6 \\
3\end{array}$ & $\begin{array}{l}(4 \cdot 5) \\
(43) \\
(39) \\
(9) \\
(4 \cdot 5)\end{array}$ & $\begin{array}{r}6 \\
24 \\
27 \\
6 \\
1\end{array}$ & $\begin{array}{l}(9 \cdot 5) \\
(37 \cdot 5) \\
(42) \\
(9 \cdot 5) \\
(1 \cdot 5)\end{array}$ \\
\hline
\end{tabular}

${ }^{\star}$ Max extension in hours.

Table 3 Initial visual acuities (VA)

\begin{tabular}{llllll}
\hline & \multicolumn{2}{c}{ Group 1 $(n=65)$} & & \multicolumn{2}{c}{ Group 2 (n=64) } \\
\cline { 2 - 3 } Initial VA & $n$ & $(\%)$ & & $n$ & $(\%)$ \\
\hline $20 / 400-20 / 500$ & 10 & $(15 \cdot 5)$ & & 9 & $(14)$ \\
20/700-20/1000 & 18 & $(27 \cdot 5)$ & & 18 & $(28)$ \\
CF/HM & 28 & $(43)$ & & 26 & $(40)$ \\
LP & 9 & $(14)$ & & 11 & $(17)$ \\
\hline
\end{tabular}


underwent vitrectomy for proliferative diabetic retinopathy or uveitis.

In all eyes primary intervention included an encircling band, when not placed previously: pre-existing radial buckles were sometimes removed. Phacoemulsification was performed if required because of lens opacification or the presence of anterior PVR. The lens capsule was completely removed with the forceps. Posterior vitrectomy via pars plana was followed by membrane peeling, with or without perfluorocarbon tamponade, anterior vitrectomy under scleral depression, and silicone oil exchange. Subretinal strands were present in a comparable percentage of cases in both groups (about 20\%) and were removed through retinotomies. In all eyes an endolaser coagulation was performed around the tears and on the encircling band and completed postoperatively when necessary. In all aphakic eyes an inferior iridectomy was made. The second and third operations consisted of lens removal, revision vitrectomy, membrane peeling under or without perfluorocarbons and additional laser treatment. All reoperations were performed within 4 weeks. In one eye a perforating corneal graft due to a corneal scar after an injury was transplanted. All surgery was performed by one experienced surgeon (SB).

No attention was given to differences between perfluorodecalin and -octane; both products (Adatomed, Germany) were used periodically, showed the same ability to flatten the retina, and were easily visible.

Postoperatively the eyes were followed over a period of 11 to 28 months (average $17 \cdot 4$ months) in group 1 and 10 to 38 months (average 24.4 months) in group 2. For the postoperative assessment of the retina the following criteria were set up:

- total retinal reattachment anterior and posterior of the encircling band

- anterior reproliferation on the buckle or posterior fibrosis without traction retinal redetachment centrally to the buckle

- anterior, posterior, or massive diffuse reproliferation with redetachment indicating reoperation

- residual elevation of the retina as a sign of persistent proliferation with or without progressive redetachment

- time of appearance and severity of reproliferation

- postoperative complications such as secondary glaucoma and bullous or band keratopathy

- functional success defined at least as doubling of the spatial resolution.

Table 4 Anatomical results in group 1 (with perfluorocarbons)

\begin{tabular}{llllll}
\hline & \multicolumn{2}{l}{$\begin{array}{l}\text { First procedure } \\
\text { (65 eyes) }\end{array}$} & & \multicolumn{2}{l}{$\begin{array}{l}\text { Second procedure } \\
(21 \text { eyes) }\end{array}$} \\
\cline { 2 - 3 } \cline { 5 - 6 } & $n$ & $(\%)$ & & $n$ & $(\%)$ \\
\hline Attached & 47 & $(72 \cdot 5)$ & & 16 & $(76)$ \\
Remained attached & 22 & $(34)$ & 10 & $(47 \cdot 5)$ \\
Elevated & 18 & $(27 \cdot 5)$ & 5 & $(24)$ \\
Reproliferation & 43 & $(66)$ & & 11 & $(52 \cdot 5)$ \\
Reoperation & 21 & $(32)$ & & $(9 \cdot 5)$ \\
Uncured & 4 & $(6)$ & & 2 & $(9 \cdot 5)$ \\
\hline
\end{tabular}

\section{Results}

GROUP 1 (WTTH PERFLUOROCARBONS) (TABLE 4) After the first operation 47 of 65 eyes (72.5\%) had postoperative total retinal reattachment; 22 of these $(47 \%)$ remained attached during the whole observation period and 25 eyes (53\%) developed reproliferation. In 15 out of 25 eyes the indication for reoperation was given. Eight eyes remained unchanged with a localised elevation of the retina. Two patients refused further surgery.

In 18 eyes in group $1(27.5 \%)$ in the retina could not be flattened completely in spite of intense surgery; six of these underwent reoperation because of increasing reproliferation. In eight eyes the retinal situation did not worsen during the follow up period. Further surgical treatment of four eyes had to be abandoned because of massive contraction of the retina with secondary holes.

In summary, 21 eyes in group 1 (32\% of the total 65 cases) needed revision vitrectomy with membrane peeling. In one eye an additional radial buckle was placed. No lens removal was necessary as even clear lenses were removed in the first step in cases of anterior proliferation. The retina of 16 of these 21 eyes $(76 \%)$ could be reattached intraoperatively, 10 of which remained attached during the observation period. Six eyes showed reproliferation which indicated the need for a third intervention in two cases, in the others $(n=4)$ the retina showed only distortion but no detachment.

Five of 21 eyes ( $21 \%$ ) had a circumscribed retinal elevation at the end of surgery. Two of these were judged as uncured cases because of diffuse reproliferation and massive traction detachment; in a third intervention which was necessary in two eyes could be treated successfully. In one eye the retina remained attached, the other one had to be left after repeated diffuse traction had developed.

In summary, a $69 \%$ success rate was achieved in group 1 .

\section{GROUP 2 (WITHOUT PERFLUOROCARBONS) \\ (TABLE 5)}

In 48 out of 64 eyes (75\%) retinal reattachment could be achieved with one operation, 21 of these $(44 \%)$ remained unchanged, 27 eyes $(56 \%)$ developed reproliferation. In 19 eyes an indication for reoperation was given, two patients refused further surgery because visual deterioration was not noticed.

In 16 of 64 cases (25\%) the retina could not be flattened totally during the first operation.

Table 5 Anatomical results in group 2 (without perfluorocarbons)

\begin{tabular}{llllll}
\hline & \multicolumn{2}{l}{$\begin{array}{l}\text { First procedure } \\
\text { (64 eyes) }\end{array}$} & & \multicolumn{2}{l}{$\begin{array}{l}\text { Second procedure } \\
\text { (23 eyes) }\end{array}$} \\
\cline { 2 - 3 } \cline { 5 - 6 } & $n$ & $(\%)$ & & $n$ & $(\%)$ \\
\hline Attached & 48 & $(75)$ & & 11 & $(48)$ \\
Remained attached & 21 & $(33)$ & & $(35)$ \\
Elevated & 16 & $(25)$ & & 12 & $(52)$ \\
Reproliferation & 43 & $(67 \cdot 5)$ & & 15 & $(65)$ \\
Reoperation & 23 & $(36)$ & & 3 & $(13)$ \\
Uncured & 10 & $(15 \cdot 5)$ & & 3 & $(13)$ \\
\hline
\end{tabular}


In the postoperative course we observed massive reproliferation in 10 of these eyes that had to be left uncured.

In the second step 23 eyes in group 2 underwent membrane peeling, in four eyes lens removal was performed, in one eye a corneal graft was transplanted.

Postoperatively 11 of the 23 eyes (48\%) showed retinal reattachment, eight of those remained totally attached. In three eyes anterior reproliferation developed but none of these required a third operation. Twelve of the 23 eyes $(52 \%)$ could not be reattached completely during surgery. An indication for membrane peeling was given in three of these cases. Six eyes with reproliferation remained unchanged without any additional surgery. Three eyes with massive diffuse reproliferation and consecutive traction detachment in the inferior half of the fundus were classified as uncured cases. Even with a third intervention two of the three eyes could not be healed; in one eye the retina was flattened finally.

To summarise, a $61 \%$ success rate was achieved in this group.

\section{APPEARANCE AND SEVERITY OF REPROLIFERATION}

In both groups we observed reproliferation not earlier than 1 month postoperatively. In group 1 the time of appearance ranged between 1 and 9 months after surgery with an average of $2 \cdot 77$ months which is comparable with that in group 2 where reproliferation started 1 to 12 months postoperatively (average 3.34 months). Anterior reproliferation on the encircling band or posterior reproliferation behind it did not develop in any specific period.

However, the rate of massive, severe reproliferation followed by anterior segment complications and extremely poor visual outcome was 2.5 times higher $(23 \%$ as against $9 \%)$ in eyes operated on without perfluorocarbon liquids (see Tables 4 and 5). We observed in our series that the use of heavy liquids improved the prognosis of eyes with postoperative reproliferation by reducing the severity.

\section{POSTOPERATIVE COMPLICATIONS}

We also investigated the benefit of intraoperative perfluorocarbon tamponades with regard to the development of secondary glaucoma and bullous or band keratopathies. Secondary glaucoma was classified as treatable when topical therapy given for several months postoperatively was sufficient to normalise the intraocular pressure, whereas in cases of untreatable glaucoma the pressure was elevated to more than $20 \mathrm{~mm} \mathrm{Hg}$ in spite of local and systemic antiglaucomatous therapy.

In group 1, in 24 of 65 eyes (37\%) the intraocular pressure increased postoperatively. Seventeen eyes $(26 \%)$ could be treated by the local application of eyedrops, seven eyes $(11 \%)$ were resistant to any therapy. In most of the cases glaucoma developed after the first operation (18 eyes), with an additional six cases of glaucoma developing after the second operation.

In the second group, 38 of 64 eyes $(59.5 \%)$ showed elevated pressure in the postoperative period. In 25 eyes (39\%) pressure could be kept normal with topical therapy; an additional 13 eyes (20\%) developed glaucoma which was classified as untreatable. Two thirds of these eyes were cases with unsuccessful surgery and contraction of the retina as a result of severe reproliferation with silicone prolapsing into the anterior chamber. The results were not statistically significant (Fisher's exact test, $\mathrm{p}=0.087$ ).

We observed a bullous or even band keratopathy caused by a long standing silicone oil tamponade in four out of the 65 eyes $(6 \cdot 1 \%)$ in group 1. Two cases developed after the first and the second operation respectively. In group 2 we found these complications in 12 out of 64 eyes $(18 \cdot 75 \%)$ always combined with secondary glaucoma. Anterior segment damage was attributed to the increased severity of reproliferation in this group. One third (four eyes) was found after the first surgery, two thirds (eight eyes) were observed after the second intervention. The complication rate has decreased significantly since the use of perfluorocarbons (Fisher's exact test, $p=0.05$ ).

\section{FUNCTIONAL RESULTS}

Preoperative visual acuities were comparable in both groups and are listed in Table 3. More than half of the patients had visual function of counting fingers or less. A postoperative improvement of vision corresponded to a doubling of the spatial resolution defined by the Retina Foundation.

At the end of follow up in group 1 visual improvement was achieved in 42 cases $(65 \%)$, 17 of those had visual acuity better than $20 / 200$. Ambulatory vision of at least $5 / 200$ was obtained in 45 eyes $(69 \cdot 5 \%)$. Visual acuity remained unchanged in 15 eyes $(23 \%)$ or deteriorated in eight eyes $(12 \%)$, three of those having no light perception. Reasons for failure were retinal redetachment, corneal haziness, or phthisis of the globe.

In group 2 visual improvement was achieved in 35 eyes (55\%), 13 out of 35 obtained 20/200 or better. Visual acuity remained unchanged in 19 cases $(29.5 \%)$ or deteriorated in 10 eyes $(15.5 \%)$, including five cases without light perception. Ambulatory vision was found in 37 eyes $(58 \%)$ at the end of follow up.

The average visual acuity of the most recent controls was higher in group $1(20 / 200)$ than in group 2 (20/400).

\section{Discussion}

The temporary stabilisation of the posterior retina by heavy perfluorocarbon liquids is an effective tool in vitreoretinal surgery. Perfluorocarbons give relief of anterior retinal traction and avoid the need for posterior retinotomies to be performed for internal drainage. ${ }^{124}$ However, proliferative vitreoretinopathy has been recognised to be a cell 
mediated process causing contraction of the peripheral vitreous gel and the formation of epiretinal and subretinal membranes. Successful surgery on retinal detachment with PVR requires treatment of both the rhegmatogenous and tractional components and avoidance of clinically significant recurrent proliferation. ${ }^{5}$ The reason for this study was how this intraoperative tool affects the postoperative anatomical and functional results and complication rates when comparing two groups of eyes with similar indications and PVR grades. A prospective randomised study would not be performed voluntarily.

The anatomical results, in all aspects, were significantly improved as might be expected. With one or more vitreoretinal operations we obtained a reattachment rate of $69 \%$ with the help of perfluorocarbons compared with $61 \%$ when they were not used. This anatomical success is in accordance with most of the results previously reported ${ }^{4-13}$ ranging from $60 \%{ }^{9}$ to $81 \%{ }^{12}$ depending on the length of observation. In all series except one ${ }^{11}$ and ours, patients with ocular trauma, eyes with a bad prognosis, ${ }^{14}$ were excluded. This group of eyes demonstrated particularly the benefit of perfluorocarbons. The haemostatic effect of heavy liquids in cases of intense bleeding during surgery was of additional advantage. A final success rate of $63 \%$ in group 1 as against $40 \%$ in group 2 was achieved but the small number of cases does not allow statistical analysis.

Separating persistent traction in cases of postoperative elevation from new periretinal proliferation in eyes with postoperative attachment, we found that in group $153 \%$ of the eyes with primary completely attached retinas developed epiretinal membranes after one operation in comparison with $56 \%$ of those eyes operated on without perfluorocarbons. Sixty per cent of these cases with perfluorocarbons as against $70.5 \%$ without perfluorocarbons had to undergo further operations. All eyes with postoperative retinal elevation (23 cases in group 1, 30 cases in group 2) developed epiretinal membranes in their later phase. In about one quarter of both groups the indication for reoperation was given. However, it is noteworthy that 15 of those 30 eyes $(50 \%)$ where surgery was performed without perfluorocarbons resulted in such a massive traction redetachment that, in agreement with the patients, we desisted from another intervention. The numbers of reproliferating cases in both groups correspond well to the findings of Lewis ${ }^{16}$ and $\operatorname{Cox}^{8}$ but do not compare with other reports ${ }^{67}$ where no differentiation between persisting and new proliferation was made.

The difference we observed in the reattachment rate after second and third interventions is remarkable but not significant $(p=0 \cdot 18$, Fisher's exact test); 18 of 23 eyes ( $79 \%$ ) could be treated successfully with the help of perfluorocarbons, 11 of these remained attached. In contrast, in only 12 of 26 reoperated cases $(46 \%)$ could the retina be flattened without using perfluorocarbons. Previously published data about surgery of recurrent PVR range between $39 \%{ }^{8}$ and $86 \% .^{15}$

We agree with other authors ${ }^{12}{ }^{15-17}$ that the development of reproliferating tissue occurs in the early postoperative period. In our series it appeared in a similar average time after surgery (2.87 months and 3.14 months respectively) in both groups. The longer follow up period of the patients who had surgery before the perfluorocarbons era cannot influence the reproliferation rate decisively as the minimum observation period was 10 months for all patients - a time during which $97 \%$ of the proliferations had already developed.

In accordance with previous reports ${ }^{12} 16$ we could identify reproliferation which started with massive contraction of the anterior retina and resulted in anterior and posterior traction redetachment as the-most common cause of anatomical failure mainly in eyes operated on unsuccessfully without perfluorocarbons. In eyes with primarily reattached retina we observed the posterior retina to be the most frequent location for the development of reproliferation in both groups (about $50 \%$ ).

This higher incidence of eyes that had to be abandoned in group 2 also explains the higher frequency of secondary glaucoma and keratopathy in this group. Temporary elevated pressure was measured in $26 \%$ in group 1 as against 39\% in group 2 . At the end of follow up chronic glaucoma was observed in $11 \%$ of the group operated on with perfluorocarbons and $20 \%$ of the group that had surgery without perfluorocarbons. The incidences reported elsewhere vary from $7 \%$ to $22 \% \%^{78101317-21}$ and refer to different observation periods and vitrectomy techniques including an inferior iridectomy in most of the studies.

A statistically significant improvement affects the number of cases of keratopathies due to silicone oil tamponade. In $6 \cdot 1 \%$ of the eyes operated on with perfluorocarbon/silicone exchange as against $18.75 \%$ of the eyes that had no perfluorocarbon tamponading band bullous keratopathy was present $(p=0.05$, Fisher's exact test). Previously reported complication rates $^{7810131719}$ range from $10 \%{ }^{19}$ to $43 \%^{10}$ depending on the presence of an inferior iridectomy, the exclusion of trauma cases, and the length of follow up.

In group 1 the anatomical success (69\%) corresponds well with the functional success rate $(69.5 \%)$ which is also comparable with other reports. ${ }^{11} 13$ In group 2 we found a 5.5\% difference between the reattachment rate $(61 \%)$ and an improvement of vision $(55.5 \%)$; the higher frequency of secondary glaucoma with consecutive optic nerve atrophy and keratopathy as well as the longer and more complicated surgery without perfluorocarbons seem to be responsible. A toxic effect of silicone oil has not yet been proved. ${ }^{21}$

WHAT ARE THE MAIN FEATURES IN OUR COMPARATIVE STUDY?

The results we obtained with one surgical intervention concerning postoperative reattachment and elevation, reproliferation and reoperations 
are comparable in nearly all aspects. However, the number of cases with massive reproliferation in postoperatively elevated retinas, leading to a rapid contraction of the retina with creation of new breaks or reopening of old ones and membrane formation, is more than twice as high in the group operated on without perfluorocarbons than in the group operated on with perfluorocarbons $(p=0.083$, Fisher's exact test).

Although the improvement of the final anatomical and functional success rate since the use of perfluorocarbons did not turn out to be statistically significant for a total of 129 cases we preferred to select a series of two homogeneous groups with comparable number of cases with the same indications for surgery that were operated with the same surgical technique performed by one experienced surgeon. The advantages of perfluorocarbons are established in facilitating vitreoretinal interventions leading to a decreased severity of postoperative reproliferation and shortening of the duration of surgery. They do not treat PVR processes pharmacologically but they have been an important step forward in the surgical management of the disease.

1 Chang S. Low viscosity liquid fluorochemicals in vitreous surgery. Am f Ophthalmol 1987; 103: 38-43.

2 Binder S, Velikay M, Wedrich A, Stolba U, Datlinger P. Die klinische Anwendung flüssiger Perfluorocarbone in de Netzhautchirurgie. Spektrum Augenheilkd 1992; 6: 4-7.

3 Machemer R, Aaberg TM, MacKenzie Freeman H, Irvine AR, Lean JS, Michels RM. An updated classification of the retinal detachment with proliferative vitreoretinopathy. Am f Ophthalmol 1991; 112: 159-65.

4 Change S, Ozmert E, Zimmerman NJ. Intraoperative Perfluorocarbon liquids in the management of proliferative vitreoretinopathy. Am $f$ Ophthalmol 1988; 106 668-74.
5 Michels RG. Surgery of retinal detachment with proliferative vitreoretinopathy. Retina 1984; 4: 63-83.

6 Gonvers $M$. Temporary silicone oil tamponade in the management of retinal detachment with proliferative management of retinal detachment with proliferative

7 McCuen BW II, Landers MB III, Machemer R. The use of silicone oil following failed vitrectomy for retinal detachment with advanced proliferative vitreoretinopathy. Ophthalmology 1985; 92: 1029-33.

8 Cox MS, Trese MT, Murphy PL. Silicone oil for advanced proliferative vitreoretinopathy. Ophthalmology 1986; 93: 646-50.

9 Stern WH, Johnson RN, Irvine AR Extended retina tamponade in the treatment of retinal detachment with proliferative vitreoretinopathy. Br f Ophthalmol 1986; 70; proliferati

10 Sell CH, McCuen BW II, Landers MB III, Machemer R. Long-term results of successful vitrectomy with silicon oil after advanced proliferative vitreoretinopathy. $A m \mathcal{F}$ Ophthalmol 1987; 103: 24-8.

11 Lucke $\mathrm{KH}$, Foerster $\mathrm{MH}$, Laqua $\mathrm{H}$. Long term results of vitrectomy and silicone oil in 500 cases of complicated retinal detachments. Am 7 Ophthalmol 1987; 104: 624-33.

12 Lewis H, Aaberg TM, Abrams GW. Causes of failure after initial vitreoretinal surgery for severe proliferative vitreoinitial vitreoretinal surgery for severe proliferative

13 Yeo JH, Glaser BM, Michels RG. Silicone oil in the treatment of complicated retinal detachment. Ophthalmology 1987; 94: 1109-13.

14 Lucke K, Laqua H. Silicone oil in the treatment of complicated retinal detachment. Berlin, Heidelberg, New York: Springer Verlag, 1990: 39-45.

15 Lewis H, Burke JM, Abrams GW, Aaberg TM. Perisilicone proliferation after vitrectomy for proliferative vitreoretinopathy. Ophthalmology 1988; 95: 583-91.

16 Lewis $\mathrm{H}$, Aaberg TM. Causes of failure after repeat vitreoretinal surgery for recurrent proliferative vitreoretinoretinal surgery for recurrent proliferati

17 Federman JL, Schubert HD. Complications associated with the use of silicone oil in 150 eyes after retina-vitreou surgery. Ophthalmology 1988; 95: 870-6.

18 Binder S, Velikay M, Zügner M, Stolba U, Wedrich A. Zum Sekundärglaukom nach Silikonölimplantation Indikation, Technik und Ergebnisse. Spektrum Augenheilkd 1988; 2: 224-7.

19 Lucke K, Laqua H. Silicone oil in the treatment of complicated retinal detachment. Berlin, Heidelberg, New York: Springer Verlag, 1990: 62-70.

20 Dimopoulos S, Heimann K, Spätkomplikationen nach Silikonölinjektion. Klin Monatsbl Augenheilkd 1986; 189: 223-7.

21 Chan C, Okun E. The question of ocular tolerance to intravitreal liquid silicone. Ophthalmology 1986; 93: 651-60 